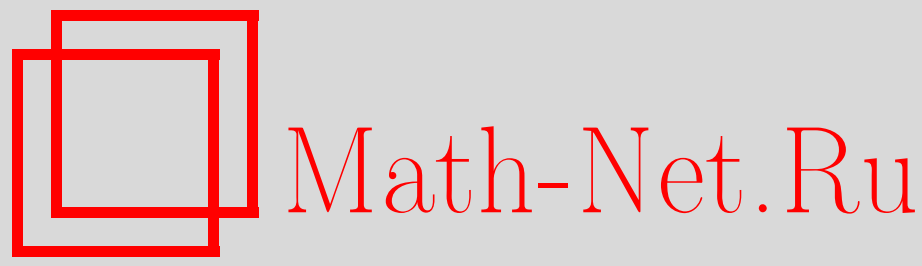

Ю. М. Зиновьев, Гравитация с точки зрения конструктивного подхода, ТМФ, 2017, том 191, номер 2, 212-218

DOI: https://doi.org/10.4213/tmf9183

Использование Общероссийского математического портала Math-Net.Ru подразумевает, что вы прочитали и согласны с пользовательским соглашением http://www . mathnet.ru/rus/agreement

Параметры загрузки:

IP: 54.224 .187 .69

26 апреля 2023 г., $17: 46: 20$

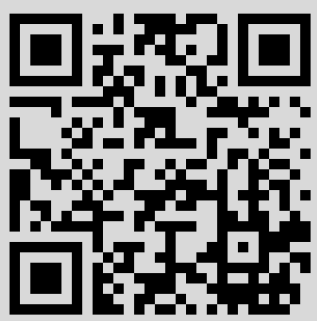




\title{
ФИЗИКА
}

Том 191, № 2

май, 2017

(C) 2017 г.

\author{
Ю. М. Зиновьев*
}

\section{ГРАВИТАЦИЯ С ТОЧКИ ЗРЕНИЯ КОНСТРУКТИВНОГО ПОДХОДА}

Общая теория относительности изначально была сформулирована как геометродинамика, т. е. теория, в которой динамической величиной является метрика пространства-времени. Однако сегодня понятно, что с точки зрения физики частиц гравитация - это просто теория безмассовой частицы со спином 2. Показано, что многие важные свойства как самой гравитации, так и ее возможных расширений и модификаций можно понять исходя из простого физического требования правильного числа физических степеней свободы и отсутствия нефизических степеней свободы.

Ключевые слова: гравитация, супергравитация, массивная гравитация, бигравитация.

DOI: https://doi.org/10.4213/tmf9183

\section{1. КИНЕМАТИКА ЧАСТИЦЫ СО СПИНОМ 2}

Лагранжево описание массивной и безмассовой частиц со спином 2 давно и хорошо известно. В самом простом варианте используется симметричный тензор второго ранга $h_{\mu \nu}=h_{\nu \mu}$. При этом лагранжиан, описывающий свободную массивную частицу в плоском пространстве-времени Минковского, имеет вид [1]

$$
\begin{aligned}
\mathcal{L}_{0}= & \frac{1}{2}\left[\partial_{\alpha} h_{\mu \nu} \partial_{\alpha} h_{\mu \nu}-\partial_{\mu} h_{\nu \alpha} \partial_{\nu} h_{\mu \alpha}-(\partial h)_{\mu}(\partial h)_{\mu}+2(\partial h)_{\mu} \partial_{\mu} h-\partial_{\mu} h \partial_{\mu} h\right]- \\
& -\frac{m^{2}}{2}\left[h_{\mu \nu}^{2}-h^{2}\right],
\end{aligned}
$$

где $(\partial h)_{\mu}=\partial^{\nu} h_{\mu \nu}, h=h_{\mu}^{\mu}$. Структура лагранжиана, в том числе конкретный вид массовых членов, полностью фиксируется требованием, чтобы из лагранжевых уравнений следовали связи (которые в дальнейшем мы будем называть векторной и скалярной связями)

$$
m^{2}\left[(\partial h)_{\mu}-\partial_{\mu} h\right]=0, \quad m^{4} h=0 .
$$

* Институт физики высоких энергий, НИЦ "Курчатовский институт", Протвино, Московская обл., Россия. E-mail: yurii.zinoviev@ihep.ru 
Эти связи гарантируют правильное число физических степеней свободы, соответствующее массивной частице со спином 2. В четырехмерном случае это пять степеней свободы, соответствующие спиральностям $\pm 2, \pm 1,0$.

Как следует из явного вида связей, в безмассовом случае обе связи теряются, однако лагранжиан становится инвариантным относительно локальных калибровочных преобразований:

$$
\delta h_{\mu \nu}=\partial_{\mu} \xi_{\nu}+\partial_{\nu} \xi_{\mu}
$$

Эта калибровочная инвариантность и гарантирует правильное число степеней свободы в безмассовом случае (при $d=4$ это спиральности \pm 2 ).

\section{2. БЕЗМАССОВАЯ ГРАВИТАЦИЯ}

Как уже отмечалось, в безмассовом случае именно калибровочная инвариантность гарантирует правильное число физических степеней свободы (и отсутствие нефизических степеней свободы). Многие важнейшие свойства гравитации можно понять, пусть и апостериори, исходя из единственного требования сохранения (хотя и модифицированной) калибровочной инвариантности после включения взаимодействия. Наиболее эффективный способ увидеть это - использовать так называемый конструктивный подход [2], [3], широко применяемый при исследовании взаимодействий в калибровочных теориях, особенно в теории полей с высшими спинами.

2.1. Конструктивный подход. Основная идея подхода, который играет роль своего рода теории возмущений для исследования взаимодействий, состоит в предположении о том, что полный нелинейный лагранжиан представляется в виде ряда по степеням полей:

$$
\mathcal{L}=\mathcal{L}_{0}+\mathcal{L}_{1}+\mathcal{L}_{2}+\cdots
$$

где $\mathcal{L}_{0}$ - свободный лагранжиан, квадратичный по полям, член $\mathcal{L}_{1}$ содержит все кубические по полям члены и т. д. Соответственно предполагается, что и калибровочные преобразования в нелинейной теории представляются в виде ряда по степеням полей:

$$
\delta=\delta_{0}+\delta_{1}+\delta_{2}+\cdots,
$$

где $\delta_{0}-$ неоднородные члены, $\delta_{1}$ - линейные по полям члены и т. д. Тогда условие калибровочной инвариантности принимает вид

$$
\begin{aligned}
\delta_{0} \mathcal{L}_{0} & =0, \\
\delta_{0} \mathcal{L}_{1}+\delta_{1} \mathcal{L}_{0} & =0, \\
\delta_{0} \mathcal{L}_{2}+\delta_{1} \mathcal{L}_{1}+\delta_{2} \mathcal{L}_{0} & =0, \quad \ldots .
\end{aligned}
$$

Первое уравнение означает, что исходный свободный лагранжиан обладает калибровочной инвариантностью. Тогда второе уравнение позволяет определить наиболее общий вид кубических вершин и т. д.

2.2. Самодействие безмассовой частицы со спином 2. В этом разделе мы обсудим применение конструктивного подхода к самодействию безмассовой частицы со спином 2. Единственное ограничение - отсутствие высших производных в лагранжиане (которое в принципе можно ослабить). Из лоренц-инвариантности следует, 
что в линейном приближении структура кубической вершины имеет вид (схематически)

$$
\mathcal{L}_{1} \sim \kappa h \partial h \partial h
$$

Отсюда видно, в частности, что константа взаимодействия с необходимостью должна быть размерной $(\kappa \sim 1 / M$ при $d=4)$. Калибровочная инвариантность требует также введения поправок к калибровочным преобразованиям вида

$$
\delta_{1} h \sim h \partial \xi \oplus \partial h \xi
$$

Важное условие - замыкание алгебры калибровочных преобразований:

$$
\left[\delta\left(\xi_{1}\right), \delta\left(\xi_{2}\right)\right]=\delta\left(\xi_{3}\right) \text {. }
$$

Результат (с точностью до тривиальных замен переменных) оказывается однозначным:

$$
\delta_{1} h_{\mu \nu}=\kappa\left[\xi^{\alpha} \partial_{\alpha} h_{\mu \nu}+\partial_{\mu} \xi^{\alpha} h_{\alpha \nu}+\partial_{\nu} \xi^{\alpha} h_{\alpha \mu}\right]
$$

Подчеркнем здесь, что, несмотря на очевидную (и неслучайную) схожесть этой формулы с общекоординатными преобразованиями тензорного поля второго ранга, в данном случае это просто калибровочные преобразования нашего поля.

Полный лагранжиан оказывается существенно нелинейным и сводится к бесконечному ряду по степеням полей:

$$
\mathcal{L} \sim \partial h \partial h \oplus h \partial h \partial h \oplus h^{2} \partial h \partial h+\cdots
$$

Тем не менее удалось показать [4], что с точностью до тривиальных замен переменных решение единственное. Более того, если ввести эффективную метрику

$$
g_{\mu \nu}=\eta_{\mu \nu}+\kappa h_{\mu \nu}, \quad g^{\mu \nu}=\eta^{\mu \nu}-\kappa h^{\mu \nu}+\cdots,
$$

где $\eta_{\mu \nu}$ - метрика пространства Минковского, то свободный лагранжиан и линейное приближение получаются при разложении в ряд по полям хорошо известного выражения:

$$
\frac{1}{\kappa^{2}} \sqrt{-g} g^{\mu \nu} \mathcal{R}_{\mu \nu}(g) \approx \mathcal{L}_{0}+\mathcal{L}_{1}+\cdots
$$

Важный и нетривиальный результат состоит в том, что в полный ответ исходная фоновая метрика $\eta_{\mu \nu}$ явно нигде не входит. Теперь в качестве фоновой метрики помимо метрики пространства Минковского (которая, конечно, является одним из решений) можно взять любое другое решение уравнений. При этом квадратичная по полям часть разложения будет описывать безмассовую частицу со спином 2 , находящуюся в пространстве, описываемом выбранной фоновой метрикой.

2.3. Взаимодействие с материей. Таким же конструктивным образом можно исследовать и вопрос о возможных взаимодействиях такой безмассовой частицы со спином 2 с материей, т. е. с частицами со спинами $s \leqslant 1$. Важный результат (который может показаться тривиальным) состоит в том, что такие взаимодействия никак не меняют структуру самой гравитации, поскольку гравитон оказывается инвариантным относительно всех внутренних симметрий, связанных с векторными полями. 
С точки зрения Стандартной модели это означает, что гравитон не имеет ни цвета, ни изоспина и гиперзаряда. Более того, если ограничиться взаимодействиями без высших производных (такие неминимальные взаимодействия возможны и могут представлять интерес, например, для космологии), то взаимодействие с полями материи

$$
\mathcal{L}=\frac{1}{\kappa^{2}} \sqrt{-g} g^{\mu \nu} \mathcal{R}_{\mu \nu}(g)+\mathcal{L}_{m}(g, \phi)
$$

с необходимостью оказывается универсальным в том смысле, что во взаимодействии фигурируют та же эффективная метрика, что и в самодействии, и та же константа.

\section{3. СУПЕРГРАВИТАЦИЯ}

Это одно из нетривиальных и перспективных расширений гравитации возникает, если рассмотреть взаимодействие гравитона и частицы со спином $3 / 2$. С точки зрения гравитации спин $3 / 2$ - это просто еше одна форма материи:

$$
\mathcal{L}=\mathcal{L}(g)+\mathcal{L}(g, \Psi)
$$

Однако поле со спином $3 / 2$ само является калибровочным полем, обладающим своими калибровочными преобразованиями со спинорным параметром. Более того, в отличие от ситуации взаимодействия с полями Янга-Миллса, гравитон уже не может быть инертным относительно таких преобразований:

$$
\delta h \sim \Psi \zeta, \quad \delta \Psi \sim \partial h \zeta
$$

Это приводит к нетривиальному расширению алгебры Пуанкаре, причем вследствие антикоммутативности фермионных полей такое расширение оказывается супералгеброй:

$$
\{Q, Q\} \sim P
$$

Одно из важнейших свойств суперсимметрии состоит в том, что она существенно улучшает ультрафиолетовое поведение гравитации и полей Янга-Миллса, что, в частности, позволяет решить проблему иерархий, которая всегда возникает в теориях с существенно разными масштабами.

С другой стороны, структура супермультиплетов (неприводимых представлений супералгебры) приводит к тому, что должны существовать суперпартнеры у всех известных частиц материи. Отсутствие вырождения бозонов и фермионов по массам и то, что такие суперпартнеры (пока) не наблюдаются, означает, что суперсимметрия должна быть нарушена, а гравитино должно иметь ненулевую массу.

\section{4. МАССИВНАЯ ГРАВИТАЦИЯ}

Важный вопрос, к которому много раз обращались в разные годы и который интенсивно обсуждается в последние годы: может ли гравитон иметь ненулевую массу? Это могло бы приводить к существенным эффектам, в первую очередь в космологии. В рамках конструктивного подхода это требует исследования самодействия массивной частицы со спином 2 и ее взаимодействия с материей. 
4.1. Самодействие массивной частицы со спином 2. Для того чтобы иметь правильное число степеней свободы, взаимодействие должно быть построено так, чтобы из лагранжевых уравнений следовали обе связи - векторная и скалярная. Есть простой способ гарантированно получить векторную связь: оставить кинетические члены такими же, как в безмассовом случае, добавив потенциал, не содержащий производных поля $h$, описывающего частицу со спином 2 :

$$
\mathcal{L}=\frac{1}{\kappa^{2}} \sqrt{-g} g^{\mu \nu} \mathcal{R}_{\mu \nu}(g)-V(\eta, h)
$$

Отметим, что потенциал уже не может быть записан с использованием только эффективной метрики, фоновая метрика будет с необходимостью явно присутствовать. В общем случае при $d=4$ в такой теории будет шесть степеней свободы - спиральности $\pm 2, \pm 1,0,0^{\prime}$, причем одна из скалярных степеней свободы будет нефизической [5]. Только недавно был найден узкий класс специального вида потенциалов, для которых скалярная связь воспроизводится [6], [7]. Замечательно, что такой потенциал может быть записан для произвольной фоновой метрики, число степеней свободы при этом правильное, однако остается открытым вопрос о том, являются ли они все физическими в случаях, когда фоновая метрика отличается от метрики пространства Минковского.

4.2. Безмассовый предел и причинность. Взаимодействия массивного гравитона с материей также можно исследовать в рамках конструктивного подхода (см., например, [8]). При этом возникает серьезная проблема, связанная с тем, что в безмассовом пределе результаты массивной теории не сводятся к результатам безмассовой теории [9], [10]. Пояснить это можно, воспользовавшись простой аналогией с электродинамикой. В массивной электродинамике (спиральности $\pm 1,0$ ) в линейном приближении фотон взаимодействует с током, построенным из полей заряженных частиц

$$
\mathcal{L}_{1} \sim A^{\mu} J_{\mu}
$$

который в безмассовом пределе должен сохраняться. При этом скалярная компонента, которая взаимодействует с дивергенцией этого тока, в безмассовом пределе отщепляется.

В массивной гравитации (спиральности $\pm 2, \pm 1,0)$ в линейном приближении гравитон взаимодействует с тензором второго ранга, построенным из полей материи:

$$
\mathcal{L}_{1} \sim h^{\mu \nu} T_{\mu \nu}
$$

который в безмассовом пределе также должен сохраняться. При этом спиральности \pm 1 , которые взаимодействуют с дивергенцией этого тензора, также отщепляются. В то же время скалярная компонента взаимодействует со следом этого тензора и безмассовый предел оказывается неуниверсальным и зависит от конкретной формы материи. Это приводит к тому, что, например, скалярная компонента дает нетривиальный вклад во взаимодействие массивных объектов (например, притяжение Земли к Солнцу), но никак не влияет на отклонение света в поле Солнца. В итоге при любой сколь угодно малой массе гравитона мы приходим к противоречию с наблюдаемыми в рамках Солнечной системы данными. 
Поскольку предыдущие рассуждения существенно опирались на результаты линейного приближения, естественно предположить, как это и сделал Вайнштейн [11], что решение может быть связано с учетом нелинейных поправок. Для компактного сферически-симметричного источника массы $M$ они могут быть существенны (т. е. экранировать вклад скалярной компоненты) при расстояниях

$$
r<R_{V} \sim\left(\frac{M}{m^{2} m_{\mathrm{Pl}}^{2}}\right)^{1 / 3}
$$

где $m$ - масса гравитона, а $m_{\mathrm{Pl}}$ - масса Планка. Однако те же самые нелинейные поправки, в свою очередь, приводят к нарушению причинности.

\section{5. БИГРАВИТАЦИЯ}

Еще один нетривиальный вопрос: может ли быть так, что в природе существует несколько гравитонов? Напомним, что аналогичный вопрос о частицах со спином 1 привел к появлению теорий Янга-Миллса, которые сегодня лежат в основе Стандартной модели.

5.1. Два (или более) безмассовых гравитона? С точки зрения конструктивного подхода это вопрос о возможных взаимодействиях в теории, содержащей два (или более) безмассовых поля $h_{\mu \nu}, f_{\mu \nu}$ со спином 2 , находящихся в некотором пространстве с фоновой метрикой $\eta_{\mu \nu}$. Если ограничиться взаимодействиями с не более чем двумя производными, то с точностью до замен переменных общее решение сводится к виду [12]-[15]

$$
\mathcal{L}=\frac{1}{k_{1}^{2}} \sqrt{-g} g^{\mu \nu} \mathcal{R}(g)+\frac{1}{k_{2}^{2}} \sqrt{-\hat{f}} \hat{f}^{\mu \nu} \mathcal{R}(\hat{f}),
$$

где

$$
g_{\mu \nu}=\eta_{\mu \nu}+k_{1} h_{\mu \nu}, \quad \hat{f}_{\mu \nu}=\eta_{\mu \nu}+k_{2} f_{\mu \nu} .
$$

Таким образом, все сводится к сумме двух невзаимодействующих между собой теорий, каждая из которых эквивалентна обычной безмассовой гравитации. Более того, при этом все поля материи также делятся на две невзаимодействующие между собой группы, каждая из которых взаимодействует только со “своей” гравитацией.

5.2. Безмассовый и массивный гравитоны. Для случая одной безмассовой и одной массивной частицы со спином 2 есть решение, гарантирующее калибровочную инвариантность для безмассовой и векторную связь для массивной частицы:

$$
\mathcal{L}=\frac{1}{k_{1}^{2}} \sqrt{-g} g^{\mu \nu} \mathcal{R}(g)+\frac{1}{k_{2}^{2}} \sqrt{-\hat{f}} \hat{f}^{\mu \nu} \mathcal{R}(\hat{f})-V(g, f)
$$

Если взять тот же потенциал, что и в массивной гравитации, с заменой фоновой метрики $\eta_{\mu \nu}$ на динамическую метрику $g_{\mu \nu}$, то есть и скалярная связь [16]. Такая теория уже нашла свое применение в космологии, однако важнейший вопрос о взаимодействии с материей остается пока открытым. 


\section{6. ЗАКЛЮЧЕНИЕ}

Теория гравитации как теория частицы со спином 2 обладает многими нетривиальными свойствами. Перечислим в заключение только некоторые из них.

1. Простое предположение о том, что существует безмассовая частица со спином 2 (гравитон), практически полностью определяет все основные особенности гравитации.

2. Невозможно построить нетривиальную теорию, в которой присутствует более чем один безмассовый гравитон.

3. Введение даже очень малой массы оказывается нетривиальной (и пока не до конца понятой) проблемой.

\section{Список литературы}

[1] M. Fierz, W. Pauli, "On relativistic wave equations for particles of arbitrary spin in an electromagnetic field", Proc. Roy. Soc. London Ser. A, 173:953 (1939), 211-232.

[2] J. Fang, C. Fronsdal, "Deformations of gauge groups. Gravitation", J. Math. Phys., 20:11 (1979), 2264-2271.

[3] K. A. Milton, L. F. Urrutia, R. J. Finkelstein, "Constructive approach to supergravity", Gen. Relat. Gravit., 12:1 (1980), 67-81.

[4] R. M. Wald, "Spin-two fields and general covariance", Phys. Rev. D, 33:12 (1986), 3613-3625.

[5] D. G. Boulware, S. Deser, "Can gravitation have a finite range?", Phys. Rev. D, 6:12 (1972), $3368-3382$.

[6] C. de Rham, G. Gabadadze, "Generalization of the Fierz-Pauli action", Phys. Rev. D, 82:2 (2010), 044020, 9 pp., arXiv: 1007.0443.

[7] C. de Rham, G. Gabadadze, A. J. Tolley, "Resummation of massive gravity", Phys. Rev. Lett., 106:23 (2011), 231101, 4 pp., arXiv: 1011.1232.

[8] Yu. M. Zinoviev, "On massive spin 2 interactions", Nucl. Phys. B, 770:1-2 (2007), 83-106, arXiv: hep-th/0609170.

[9] В.И. Захаров, “Линеаризованная теория гравитации и масса гравитона", Писъма в ЖКЭТФ, 12:9 (1970), 447-449.

[10] H. Van Dam, M. Veltman, "On the mass of the graviton", Gen. Relat. Gravit., 3:3 (1972), $215-220$.

[11] A. I. Vainstein, "To the problem of nonvanishing gravitation mass", Phys. Lett. B, 39:3 (1972), 393-394.

[12] C. Cutler, R. M. Wald, "A new type of gauge invariance for a collection of massless spin 2 fields: I. Existence and uniqueness", Class. Quantum Grav., 4:5 (1987), 1267-1278.

[13] R. M. Wald, "A new type of gauge invariance for a collection of massless spin-2 fields: II. Geometrical interpretation", Class. Quantum Grav., 4:5 (1987), 1279-1316.

[14] N. Boulanger, T. Damour, L. Gualtieri, M. Henneaux, "Inconsistency of interacting, multi-graviton theories", Nucl. Phys. B, 597:1-3 (2001), 127-171, arXiv: hep-th/0007220.

[15] N. Boulanger, "Multi-graviton theories: yes-go and no-go results", Fortschr. Phys., 50:8-9 (2002), 858-863, arXiv: hep-th/0111216.

[16] S. F. Hassan, R. A. Rosen, "Bimetric gravity from ghost-free massive gravity", JHEP, 02 (2012), 126, 11 pp., arXiv: 1109.3515. 